\section{PROFISSIONAIS DE SAÚDE DA UNIDADE DE TERAPIA INTENSIVA: PERCEPÇÃO DOS FATORES RESTRITIVOS DA ATUAÇÃO MULTIPROFISSIONAL}

\author{
Healthcare professionals of an intensive care unit: perception \\ of restrictive factors of the multiprofessional work
}

\begin{abstract}
Profesionales sanitarios de la unidad de cuidados intensivos: percepción de factores restrictivos de la actuación multiprofesional
\end{abstract}

\section{RESUMO}

Objetivo: Identificar a percepção dos profissionais de saúde de uma Unidade de Terapia Intensiva (UTI) sobre os fatores restritivos do trabalho em equipe multiprofissional. Métodos: Estudo exploratório, descritivo, com abordagem qualitativa, que ocorreu em um hospital terciário de alta complexidade, localizado em Sobral/CE. Participaram nove entrevistados representantes da equipe multiprofissional da UTI. Os dados foram coletados por entrevista semiestruturada e analisados segundo a Análise de Conteúdo. Assim, as interlocuções com os profissionais foram apreendidas em quatro categorias: desrespeito entre os profissionais da equipe, excesso de demanda, falta de comunicação entre seus integrantes e falta de capacitação profissional. Resultados: $\mathrm{O}$ fator mais restritivo do trabalho em equipe multiprofissional na UTI foi a falta de respeito entre os integrantes da equipe, resultado das relações de hierarquia de poder, da falta de conhecimento do fazer de cada profissional, bem como da falta de comunicação dentro da equipe, evidenciando a necessidade de estratégias que potencializem a comunicação e o respeito entre os integrantes. Além disso, o excesso de demanda, somando-se a um ambiente estressante como a UTI e à falta de capacitação da equipe foram destacados nas falas como fatores considerados restritivos ao processo. Conclusão: Foi possível levantar os fatores restritivos do trabalho em equipe multiprofissional no cenário da UTI, o que pode colaborar para a construção e o fortalecimento das ações para superar esses desafios. Ratifica-se a importância da compreensão de que as ações a serem desenvolvidas são da equipe multidisciplinar como um todo, e não de um indivíduo apenas.

Descritores: Unidades de Terapia Intensiva; Equipe de Assistência ao Paciente; Comunicação Interdisciplinar.

\section{ABSTRACT}

Objective: To identify the perception of health professionals of an Intensive Care Unit (ICU) regarding the restrictive factors of the work in a multiprofessional team. Methods: Qualitative descriptive exploratory study conducted in a tertiary referral hospital located in Sobral, Ceará. Participants were nine representatives of the ICU multiprofessional team. Data were collected using semi-structured interviews and underwent Content Analysis. Thus, dialogues with professionals were divided into four categories: disrespect between team professionals, excessive demand, lack of communication between members and lack of professional training. Results: The most restrictive factor of the work of the multiprofessional team in the ICU was the lack of respect between team members, which results from the hierarchy of power relationships, the lack of knowledge of each professional's work as well as the lack of communication within the team, highlighting the need for strategies to enhance communication and respect between members. In addition, the excessive demand coupled with a stressful environment such as the ICU and the lack of professional training were highlighted in the speeches as factors considered restrictive to the process. Conclusion: It was possible to identify the restrictive factors of the work in a multiprofessional team
Artigo Original
1) Hospital Estadual Dirceu Arcoverde HEDA - Parnaíba (PI) - Brasil

2) Hospital Regional Norte - HRN - Sobral (CE) - Brasil

3) Escola de Saúde Pública do Ceará - ESP/ CE - Fortaleza (CE) - Brasil
Recebido em: $12 / 11 / 2015$

Revisado em: 26/01/2016

Aceito em: 17/02/2016 
in an ICU setting, which can contribute to the construction and strengthening of actions to overcome these challenges. It is important to understand that the actions to be developed are responsibilities of the whole multiprofessional team - not just one's responsibility.

Descriptors: Intensive Care Units; Patient Care Team; Interdisciplinary Communication.

\section{RESUMEN}

Objetivo: Identificar la percepción de los profesionales sanitarios de una Unidad de Cuidados Intensivos (UCI) sobre los factores restrictivos del trabajo de equipo multiprofesional. Métodos: Estudio exploratorio, descriptivo y de abordaje cualitativo en un hospital terciario de elevada complejidad localizado en Sobral/ CE. Participaron nueve entrevistados representantes del equipo multifprofesional de la UCI. Los datos fueron recogidos a través de entrevista semiestructurada y analizados según el Análisis de Contenido. Así, las interlocuciones entre los profesionales fueron identificadas en cuatro categorías: falta de respeto entre los profesionales del equipo, exceso de demanda, falta de comunicación entre los integrantes y falta de capacitación profesional. Resultados: El factor más restrictivo del trabajo en equipo multiprofesional de la UCI ha sido la falta de respeto entre los integrantes del equipo que resulta de las relaciones de jerarquía de poder, de la falta de conocimiento, del hacer de cada profesional así como de la falta de comunicación dentro del equipo lo que evidencia la necesidad de estrategias que potencialicen la comunicación y el respecto de los integrantes. Además, el exceso de demanda asociado al ambiente de estrés de la UCI y la falta de capacitación del equipo se destacaron en los relatos como factores restrictivos del proceso. Conclusión: Fue posible identificar los factores restrictivos del trabajo de equipo multiprofesional en el escenario de la UCI lo que puede colaborar para la construcción y el fortalecimiento de las acciones para la superación de los desafios. Ratificase la importancia de la comprensión de que las acciones desarrolladas son de todo el equipo multidisciplinario y no solamente de un individuo.

Descriptores: Unidades de Cuidados Intensivos; Grupo de Atención al Paciente; Comunicación Interdisciplinaria.

\section{INTRODUÇÃO}

$\mathrm{Na}$ área da saúde, o trabalho em equipe é considerado um mecanismo indispensável na atuação dos profissionais, em contraposição ao intenso processo de especialização e fragmentação das ações geradas por esses indivíduos. Nesse contexto, a abordagem da equipe multiprofissional mostra-se como uma estratégia que pode levar a uma maior interação entre as diferentes áreas do conhecimento ${ }^{(1)}$.

No âmbito do Sistema Único de Saúde (SUS), o trabalho desenvolvido nas Unidades de Terapia Intensiva (UTI) sempre foi essencialmente concebido como ações desempenhadas em equipe multiprofissional, a priori, formada por profissionais médicos, enfermeiros e técnicos de enfermagem. Nesses serviços de saúde, tais profissionais exercem funções assistenciais, executando desde procedimentos mais simples até os que oferecem maior risco à vida do cliente, além das atividades gerenciais nos diferentes níveis de atenção à saúde ${ }^{(2)}$. Porém, vale destacar que, para oferecer o suporte necessário aos usuários dos serviços gravemente comprometidos, uma grande variedade de outros profissionais precisa estar envolvida no processo de restabelecimento de sua saúde, a exemplo: nutricionistas, psicólogos, fonoaudiólogos, farmacêuticos, assistentes sociais, dentre outros, como equipe de apoio, mas com igual importância para a assistência integral e de qualidade $^{(3)}$.

Para garantir a segurança e a redução de sofrimento do cliente e de seus cuidadores, a prática colaborativa entre os vários profissionais de saúde com diferentes experiências profissionais promove uma assistência da mais alta qualidade $^{(4)}$. Colaboração ou cooperação interprofissional se apresenta como uma estratégia do trabalho em equipe e está relacionada a uma ética do cuidado, aproximandose de práticas participativas e de relacionamentos pessoais mútuos e recíprocos entre os profissionais de saúde ${ }^{(5)}$.

Não obstante, as constantes situações de emergência exigindo rapidez de raciocínio e ação, o fato de ser uma unidade fechada e geralmente restrita aos que nela trabalham, a complexidade tecnológica e científica e o enfrentamento frequente com a morte são fatores que podem ameaçar a atmosfera desse ambiente, tornando-o mais estressante tanto para o profissional quanto para o cliente ${ }^{(6)}$.

Além disso, os trabalhadores precisam lidar com aumento da demanda de aprendizagem de novas habilidades, adaptação às diferentes formas de trabalho, exigências cada vez maiores à alta produtividade e máxima qualidade dos produtos/serviços em tempo reduzido, maior competitividade no mercado de trabalho, condições laborais precárias e menores benefícios empregatícios ${ }^{(7)}$.

Assim, a realidade vivenciada pelos profissionais que atuam em UTI é permeada por variados conflitos, sentimentos e emoções, o que requer uma excelente capacitação técnico-científica e preparo profissional e emocional; em contrapartida, exige sobretudo habilidade para gerenciar tais condições enquanto equipe multiprofissional, exercitando ao máximo o trabalho em equipe.

Em presença desse panorama, a relevância do estudo se configura diante da compreensão da realidade vivenciada pela equipe multiprofissional que atua na UTI, uma vez que facilitará a identificação dos fatores que dificultam a sua atuação, os quais podem estar contribuindo para a despersonalização do atendimento multiprofissional. 
Nesse sentido, o objetivo deste trabalho é identificar a percepção dos profissionais de saúde de uma Unidade de Terapia Intensiva (UTI) sobre os fatores restritivos do trabalho em equipe multiprofissional.

\section{MÉTODOS}

Trata-se de um estudo do tipo exploratório, descritivo, de abordagem qualitativa, que corresponde à análise das dimensões que escapam aos indicadores e expressões numéricas. As abordagens qualitativas propiciam subsídios para mudanças e transformações a partir dos dados gerados pela participação dos entrevistados envolvidos. Isso propicia, ainda, capacitar os grupos de interesse envolvidos na avaliação, aumentando seu potencial de análise para se constituírem sujeitos do processo de mudança ${ }^{(8)}$.

O cenário da pesquisa foi o Município de Sobral, localizado na mesorregião noroeste do Estado do Ceará, que constitui cenário de referência para a Região Nordeste, baseado na sua singularidade e nas inovações do seu modelo tecnoassistencial de saúde ${ }^{(9)}$.

O município conta com um hospital terciário de alta complexidade, o maior do Ceará e o maior hospital regional do interior da Região Nordeste. Possui quatro Unidades de Terapia Intensiva (UTI) - duas para adultos, uma pediátrica e uma neonatal, cada uma contando com 10 leitos, todas com uma equipe multiprofissional qualificada e de alta capacidade técnica, de suma importância para assistência integral, contínua e humanizada aos pacientes críticos internados. Optou-se por trabalhar com a UTI Adulto 01 como cenário deste estudo, por ser a UTI com maior tempo de funcionamento no hospital.

Como respondentes do presente estudo, selecionaram-se os atores implicados diretamente com o processo avaliativo em questão. Foram convidados a participar do estudo um representante de cada classe profissional integrante da equipe multiprofissional da UTI Adulto 01, a saber: médico, enfermeiro, técnico de enfermagem, fisioterapeuta, nutricionista, fonoaudiólogo, psicólogo, farmacêutico e assistente social, totalizando nove participantes.

A seleção dos entrevistados foi realizada adotandose alguns critérios básicos: de preferência os profissionais diaristas da equipe da manhã (maior tempo de trabalho em conjunto); fazer parte da equipe há pelo menos seis meses e estar em pleno exercício de suas funções durante a realização da pesquisa; concordar em participar do estudo, após serem informados dos objetivos, assinando o Termo de Consentimento Livre Esclarecido.

Como na abordagem qualitativa de pesquisa, o critério de inclusão dos respondentes não é numérico, considerandose que devem ser identificados aqueles que podem oferecer maior amplitude e mais variações do fenômeno em estudo. Dessa forma, pensou-se em critérios básicos para a seleção dos entrevistados desta investigação, considerando-se fundamental ter um conjunto diversificado de informantes para possibilitar a apreensão das semelhanças e diferenças ${ }^{(10)}$.

A técnica utilizada para coleta de dados foi a entrevista semiestruturada e individual, realizada no mês de março de 2015, utilizando uma questão norteadora: qual sua percepção sobre os fatores restritivos do trabalho em equipe multiprofissional da Unidade de Terapia Intensiva (UTI) onde atua?

As entrevistas foram gravadas e posteriormente transcritas, sendo assegurados o sigilo e o anonimato dos entrevistados. Para assegurar o anonimato, os participantes foram identificados pela letra $\mathrm{R}$ (Respondentes) e pelo número sequencial da entrevista $(1,2 \ldots)$. Por exemplo: o primeiro respondente entrevistado foi identificado como (R1), o segundo como (R2) e assim sucessivamente.

Para análise das falas, utilizou-se a Análise de Conteúdo ${ }^{(11)}$, através da qual optamos pela análise temática ou categorial. Essa análise é definida como um conjunto de técnicas de análise das comunicações que utiliza procedimentos sistemáticos e objetivos de descrição do conteúdo das mensagens em três fases: 1) pré-análise; 2) exploração do material; e 3) tratamento dos resultados, inferência e interpretação(11). Na primeira etapa, de préanálise, são elaboradas as operações preparatórias para a análise propriamente dita. É uma etapa de escolha dos documentos ou definição do corpus de análise, formulação das hipóteses e dos objetivos da análise, e elaboração dos indicadores que fundamentam a interpretação final. Segunda etapa: exploração do material ou codificação, que consiste em agregar trechos codificados em busca da compreensão. Tal agregação possibilita constituir as categorias e subcategorias. Terceira etapa: tratamento dos resultados - inferência e interpretação. Busca-se, nessa etapa, destacar as informações fornecidas pela análise, através de quantificação simples (frequência) ou mais complexa, como a análise fatorial, permitindo apresentar os dados.

Assim, as interlocuções resultantes com os profissionais foram apreendidas em quatro categorias: desrespeito entre os profissionais da equipe, excesso de demanda, falta de comunicação entre seus integrantes e falta de capacitação profissional.

O presente estudo seguiu a Resolução 466/2012(12), sendo aprovado pelo Comitê de Ética em Pesquisa da ESP/ CE, com liberação do Parecer Aprovado no 963.030, em 19 de fevereiro de 2015.

\section{RESULTADOS E DISCUSSÃO}

A seguir, serão apresentadas as categorias temáticas que emergiram do estudo. Todas elas evidenciam a restrição do trabalho em equipe multiprofissional na UTI. 


\section{Desrespeito entre os profissionais da equipe}

Essa categoria está relacionada à questão da hierarquia de poder dentro da equipe, principalmente no direcionamento das condutas terapêuticas:

\begin{abstract}
"Os que restringem às vezes são uma chamada hierarquia, não é?" (R1)

"Às vezes você dá um diagnóstico, diz uma conduta, e alguns profissionais não respeitam aquela conduta para determinado cliente." (R1)

"[...] São profissionais que de alguma forma não valorizam o conhecimento do outro profissional, como se ele fosse superior ou mais importante do que o conhecimento de outra pessoa que faz parte da mesma equipe." (R5)
\end{abstract}

A hierarquia existente entre os profissionais que constituem a equipe representa um obstáculo, na medida em que envolve a delimitação da área de atuação e dos procedimentos que podem ser executados por cada um, assim como a defesa dos espaços conquistados e dos privilégios adquiridos, podendo manifestar-se de diversas maneiras, quer sejam de forma velada ou disputas declaradas, mas geralmente resultam em variadas contestações de poder no que se refere à tomada de decisão sobre as condutas técnicas ${ }^{(13,14)}$.

Estudiosos destacam um "espaço de aparência" que se refere ao comportamento das pessoas quanto às suas posições/representações em um grupo social de convivência, podendo um indivíduo exercer o poder ou ser influenciado pelo poder de outro. Esse espaço é relacional e só existe quando os homens se reúnem, não sendo durável ou permanente. Quando ele ocorre no ambiente de saúde, mostra-se pertinente ao processo de trabalho da equipe multiprofissional, sofrendo influência da liderança política, da relação interpessoal, do acolhimento, do trabalho cotidiano, da interação profissional, das articulações entre as ações e das relações de poder entre profissionais e equipe/ cliente $^{(15)}$.

E dependendo de como estão elaborados esses espaços, surgem problemas éticos que acabam afetando de modo negativo a relação, acarretando prejuízos no cuidado prestado ao cliente, sendo necessário destinar esforços para que esses problemas sejam evitados ou amenizados ${ }^{(16)}$. Além da ética profissional, o desrespeito gera desgaste emocional representados pelo aumento das críticas entre os membros da equipe, implicâncias ou discussões ${ }^{(17)}$.

Toda essa problemática se expressa mais especificamente na UTI, pois se trata de uma unidade que, por longo período, contou apenas com os profissionais médicos e de enfermagem. Só mais recentemente, e aos poucos, as outras classes profissionais, como fisioterapeutas, nutricionistas, fonoaudiólogos, psicólogos, farmacêuticos e mais recentemente o cirurgião-dentista, dentre outras, vêm conseguindo conquistar o seu espaço dentro da equipe ${ }^{(2,3)}$.

Outro motivo para essa falta de respeito apontada pelos entrevistados foi o desconhecimento do fazer de cada categoria profissional, apontado como fator restritivo ao trabalho em equipe e o que motivou a expectativa de espaços; momentos que favoreçam essa troca de conhecimento de saberes entre os profissionais, para que cada membro da equipe possa conhecer e acatar os demais integrantes, compreendendo a importância do mesmo de forma individual e como membro da equipe.

"Muitos profissionais que fazem parte da equipe multiprofissional não se interessam por conhecer o trabalho do outro." (R5)

"Muitos colegas não têm conhecimento da importância da profissão daquele outro colega e acabam por banalizar, determinadas condutas." (R7)

As consequências desses fenômenos podem resultar no distanciamento e fragmentação da equipe, e como consequência, trazer prejuízos ao atendimento dos clientes. Assim, são importantes todos os esforços para a integração entre os profissionais e efetivação da interdisciplinaridade, o que torna o trabalho em equipe mais sistemático, eficiente e menos oneroso ${ }^{(5,14)}$.

Para que um grupo se transforme em equipe interdisciplinar, são necessários alguns fatores, dentre eles: mais intercooperação, comprometimento, desenvolvimento da própria equipe (pares e liderança) com reuniões e outras atividades (foco relacional); mais valorização profissional, como salário, plano de carreira e reconhecimento; mais reuniões da equipe com as lideranças internas; melhorias da estrutura física, tendo espaço para reuniões, insumos e estrutura para atendimentos; melhor dimensionamento do trabalho para o número de profissionais e maior conhecimento sobre o trabalho em grupo $^{(18)}$.

\section{Excesso de demanda}

Outro ponto relevante que surgiu dos relatos dos profissionais foi o excesso de demanda. Essa categoria trata principalmente da sobrecarga burocrática do processo de trabalho na UTI, um tema já bem discutido na literatura, principalmente quando da abordagem da equipe de enfermagem:

"Eu acho que o que restringe é a correria. A gente tem muitas atribuições." (R2)

"[...] A questão da sobrecarga de trabalho que atrapalha." (R2)

"[...] É justamente a correria. [...] é tudo muito burocrático em UTI, tudo a gente tem que escrever muito, são muitos protocolos." (R8) 
Deve serconsiderado que uma equipe superdimensionada resulta em alto custo na UTI; por outro lado, sabe-se que um grupo reduzido tende a determinar prejuízo na qualidade da assistência, interferindo na segurança do cliente, prolongando a internação e gerando maior custo $^{(19)}$. O ideal seria manter um equilíbrio entre as atividades relacionadas ao cuidado direto e indireto do cliente, no entanto, na realidade, a carga de trabalho nesse ambiente abrange outros fatores em que determinadas atividades não relacionadas com o cliente $\mathrm{e}$ seus familiares fazem parte do dever a ser cumprido pelos profissionais durante seu turno de trabalho ${ }^{(20)}$.

Apesar de lhes serem atribuídos cuidados de um número menor de clientes, é exigida da equipe multiprofissional da UTI a necessidade de maior acuidade, monitoração contínua e avaliação frequente de parâmetros clínicos e laboratoriais, além de outros aspectos pertinentes à assistência ao cliente grave. A incorporação de novos conhecimentos e aparatos tecnológicos na UTI tem influenciado o aumento do grau de complexidade assistencial e o nível de atenção requerido para o cuidado, o que consequentemente elevou o grau de sobrecarga de trabalho da equipe, com destaque para a equipe de enfermagem ${ }^{(21,22)}$.

Uma pesquisa feita com a equipe de enfermagem comprovou que as características associadas com aumento da carga de trabalho foram tipo de internação (cirurgia de urgência) e desfecho do cliente (não sobrevivente); já a gravidade do cliente e as disfunções orgânicas mostraram correlação moderada com a carga de trabalho da categoria ${ }^{(20)}$.

Um estudo de revisão de literatura concluiu que a elevação da carga de trabalho da enfermagem esteve associada ao aumento dos índices de infecção e de mortalidade em clientes internados em UTI, uma vez que afeta diretamente a exaustão física e emocional da equipe, interferindo na sua segurança, representada pela ocorrência de falhas assistenciais ${ }^{(23)}$. O ambiente da UTI é instável e, na maioria das vezes, os plantões transcorrem em um clima de agitação. As atividades são intensas, especialmente quando ocorre admissão de clientes muito graves, o que exige atenção e cuidado rigoroso de todos os integrantes dessa equipe multiprofissional para evitar ao máximo possíveis erros em decorrência do excesso de demando exigido nessa unidade ${ }^{(21)}$.

Diante desses quadros, as instituições e seus trabalhadores poderiam adotar estratégias de prevenção e/ ou redução do nível de estresse, minimizando o impacto causado pelo excesso da demanda ${ }^{(7)}$.

\section{Falta de comunicação entre seus integrantes}

Também foi dada ênfase neste estudo, como fator restritivo ao trabalho em equipe na UTI, a falta de comunicação entre seus integrantes. Essa categoria evidencia o que parece estar ligado ao ambiente estressante da UTI, ao excesso de demanda por parte da equipe, bem como às relações interpessoais entre seus integrantes:

"É o que eu acho que o que falta para a questão do trabalho em equipe é as pessoas aprenderem a se comunicar mais." (R2)

"Se a gente tem um cliente grave e falhar na questão da parte de comunicação da equipe, a gente pode falhar na conduta daquele cliente." (R2)

O tratamento do cliente é consequência do trabalho de toda a equipe de profissionais, assim, a comunicação e a troca de informações devem ocorrer continuamente para a melhor qualidade no cuidado. A comunicação entre os profissionais que integram a equipe multidisciplinar tornase de fundamental importância para não ocorrer distorções e falhas no atendimento, pois através dela são fornecidas informações que contribuem para o conhecimento uniforme entre os membros da equipe. Contudo, o ambiente na UTI não propicia a troca de informações, devido ao alto nível de ansiedade e tensão, quando as falhas de comunicação chegam a ser responsáveis por $32 \%$ dos erros nesse setor ${ }^{(5,23)}$.

Os profissionais precisam ter consciência de que a comunicação configura-se como um elemento essencial no cuidado. Ela é o alicerce das relações interpessoais, e o cuidado, nessa perspectiva, associa-se à prática de comunicar-se. Então, a comunicação, em suas variadas formas, tem um papel de instrumento de significância humanizadora, e, para tal, a equipe precisa estar disposta e envolvida para estabelecer essa relação ${ }^{(24)}$.

Infelizmente, a UTI não é um ambiente propício à comunicação aberta, clara e contínua, devido à sobrecarga de funções por parte da equipe, ao barulho, alarmes, contato com clientes graves, situações inesperadas, interrelacionamento constante entre as mesmas pessoas da equipe durante todo o turno, bem como à exigência excessiva de segurança, respeito e responsabilidade para com o cliente em sofrimento, dupla jornada de trabalho comum entre estes profissionais, contato com dor e morte iminente. Todos esses fatores dificultam a comunicação junto à equipe interdisciplinar, o que constitui um obstáculo à melhoria da qualidade do cuidado ${ }^{(21,25)}$. A vivência de momentos que propiciem a relação de comunicação poderia ser utilizada como estratégia de superação de algumas fragilidades da equipe.

Resultados de uma pesquisa apontaram que o processo de comunicação entre os membros da equipe de enfermagem foi otimizado em função do cuidado ao cliente através da interação humana, relação de interdependência, reconhecimento profissional, melhora do entendimento do outro, compartilhamento de informações através de reuniões em grupo, quando os assuntos couberem a todos da 
equipe, e conversas individuais e reservadas pelos membros da equipe quando necessário ${ }^{(24)}$.

Falhas de comunicação, resultado da não colaboração entre os profissionais de saúde, são a principal causa de dano acidental em todos os ambientes de cuidado em saúde, especialmente em unidade de terapia intensiva, que é um ambiente de trabalho dinâmico, complexo, expondo os clientes continuamente às complexidades do funcionamento da equipe interprofissional ${ }^{(7)}$.

\section{Falta de capacitação}

Por fim, esta categoria, para o trabalho em equipe multiprofissional, levantou a questão da ausência de treinamento e qualificação dos profissionais em serviço:

"Infelizmente, não é (sic) todos que tem perfil para trabalhar em equipe." (R2)

“[...] É pessoal, ainda. É porque a gente está fazendo a clínica, aprendendo mesmo no dia a dia. A gente não tem o conhecimento da faculdade." (R6)

"[...] Falta de afinidade que às vezes existe. A pessoa não tem tanta afinidade com a equipe, com a alta complexidade que é a UTI, então acho que acaba prejudicando e restringindo o trabalho de equipe multiprofissional." (R9)

A UTI é uma unidade que concentra profissionais especializados, uma variedade de recursos tecnológicos sofisticados e de alto custo para atender clientes em estado grave que necessitam assistência intensiva. Devido a tais características, a equipe desse setor difere de outros setores de internação porque, em geral, os profissionais devem ter conhecimento especializado e habilidades adicionais àquelas adquiridas em sua formação.

As UTI são unidades destinadas aos cuidados em situações críticas de saúde e cujos processos de trabalho exigem qualificação permanente das equipes, estimulando a reflexão constante sobre a prática e a construção do conhecimento, não apenas no âmbito da utilização de materiais, bem como de equipamentos especializados e competência técnica, científica e emocional ${ }^{(26)}$.

Vale mencionar que o trabalho em equipe representa um instrumento básico do cuidado em saúde, que demanda conhecimento técnico, científico e administrativo, capacidade de adaptações e mudanças, criatividade e espírito de inovação e facilidade de relacionamento interpessoal. Nesse sentido, a formação de recursos humanos com habilidades diferenciadas é essencial. Todavia, a formação acadêmica dos profissionais muitas vezes não contribui de forma direta para o desenvolvimento dessas habilidades. O profissional deve recriar o seu próprio trabalho a partir da inserção em uma rede de relações de poderes, saberes e interesses para se situar efetivamente em uma equipe ${ }^{(13,14)}$.
Por conseguinte, compreende-se que a qualificação dos profissionais e a produção de conhecimento devem fazer parte de um processo contínuo e permanente, de disseminação de informações e avaliação dos recursos humanos. Em função da alta tecnologia e cuidados de maior complexidade técnica, a educação continuada, bem como a educação permanente nas UTI, configuram-se como um desafio para os profissionais da equipe ${ }^{(26)}$.

\section{CONSIDERAÇÕES FINAIS}

A partir da opinião dos entrevistados envolvidos na presente pesquisa, foi possível identificar que o fator mais restritivo do trabalho em equipe multiprofissional na UTI foi o desrespeito entre os integrantes da equipe, resultado das relações de hierarquia de poder, bem como da falta de conhecimento do fazer de cada profissional dentro da equipe, assim como a falta de comunicação dentro da equipe, evidenciando a necessidade de estratégias que potencializem a comunicação, bem como o respeito entre os integrantes da equipe.

Além disso, o excesso de demanda, somando-se a um ambiente estressante como a UTI, e a falta de Capacitação da equipe foram destacados nas falas como fatores considerados restritivos ao processo.

Uma das limitações do presente estudo é que ele teve como objeto de investigação a Unidade Terapia Intensiva de um hospital específico, entretanto, vale ressaltar que a pesquisa envolveu uma variedade de categorias profissionais integrantes da equipe da multiprofissional, englobando a equipe básica obrigatória e os profissionais apoiadores, o que diferencia o estudo da maioria dos achados na literatura, os quais geralmente são trabalhos abordando a equipe básica, mais especificamente a equipe de enfermagem. Também foi possível levantar os pontos relevantes sobre os fatores restritivos do trabalho em equipe multiprofissional neste cenário o que pode colaborar para construção e fortalecimento das ações para superar esses desafios.

Destarte, acreditamos que seja importante mais estudos voltados para a abordagem de todos os profissionais integrantes da equipe multiprofissional da UTI, considerando todas as peculiaridades e os reflexos desse ambiente na atuação de seus profissionais, cujas relações interpessoais precisam ser consideravelmente preservadas, haja vista a complexidade dos procedimentos a serem realizados e as conflitantes tomadas de decisões às quais os profissionais se veem expostos diariamente. Ratificamos a importância da compreensão de que as ações a serem desenvolvidas são da equipe multidisciplinar como um todo, e não de um indivíduo apenas.

\section{REFERÊNCIAS}

Rev Bras Promoç Saúde, Fortaleza, 29(1): 43-50, jan./mar., 2016 
1. Peduzzi M. Equipe multiprofissional de saúde: conceito e tipologia. Rev Saúde Pública. 2001;35(1):103-9.

2. Purin N, Puri V, Dellinger RP. History of technology in the intensive care unit. Crit Care Clin. 2009;25(1):185200 .

3. Ministério da Saúde (BR), Agência Nacional de Vigilância Sanitária - ANVISA. Resolução - RDC $\mathrm{n}^{\mathrm{o}}$ 7. Dispõe sobre os requisitos mínimos para funcionamento de Unidades de Terapia Intensiva e dá outras providências. Brasília: ANVISA; 2010.

4. Rose L. Interprofessional collaboration in the ICU: how to define? Nurs Crit Care. 2011;16(1):5-10

5. Pirolo SM, Ferraz CA, Gomes R. A integralidade do cuidado e ação comunicativa na prática interprofissional da terapia intensiva. Rev Esc Enferm USP. 2011;45(6):1396-402.

6. Leite MA, Vila VSC. Dificuldades vivenciadas pela equipe multiprofissional na Unidade de Terapia Intensiva. Rev Latinoam Enferm. 2005;13(2):145-50.

7. InoueI KC, Versa GLGS, Murassaki ACM, Melo WA, Matsuda LM. Estresse ocupacional em enfermeiros intensivistas que prestam cuidados diretos ao paciente crítico. Rev Bras Enferm. 2013;66(5):722-9

8. Bosi MLM, Mercado FJ. Avaliação qualitativa de programas de saúde: enfoques emergentes. Petrópolis: Vozes; 2006.

9. Linhares JH, Pinto PD, Albuquerque IMN, Freitas CASL. Análise das ações da Fisioterapia do NASF através do SINAI no município de Sobral - CE. Cad Escola de Saúde Pública. 2010;4(2):32-41.

10. Minayo MCS. O desafio do conhecimento: pesquisa qualitativa em saúde. 9a ed. São Paulo: Hucitec; 2006.

11. Bardin L. Análise de conteúdo. Trad. Luís Antero Reto e Augusto Pinheiro. Lisboa. Edições 70; 2010.

12. Ministério da Saúde (BR), Conselho Nacional de Saúde (CNS), Comissão Nacional de Ética em Pesquisa. Resolução $n^{\circ}$ 466/2012. Normas regulamentadoras de pesquisa envolvendo seres humanos. Brasília: CNS; 2012.

13. Oliveira EB, Silva AV, Perez EF Junior, Costa HF, Nascimento LP, Souza LAM. Fatores de risco psicossocial em terapia intensiva neonatal: repercussões para a saúde do enfermeiro. Rev Enferm UERJ. 2013;21(4):490-5.

14. Peres RS, Anjos ACY, Rocha MA, Guimarães AGC, Borges GM, Souza KG et al. O Trabalho em Equipe no
Contexto Hospitalar: reflexões a partir da experiência de um programa de residência multiprofissional em saúde. Em Extensão (Uberlândia, MG). 2011;10(1):113-20.

15. Oliveira HM, Moretti-Pires RO, Parente Rosana Cristina Pereira. As relações de poder em equipe multiprofissional de Saúde da Família segundo um modelo teórico arendtiano. Interface Comun Saúde Educ. 2011;15(37):539-50.

16. Amestoya SC, Backesb VMS, Thofehrnc MB, Martinid JG, Meirellese BHS, Trindadef LL. Gerenciamento de conflitos: desafios vivenciados pelos enfermeiroslíderes no ambiente hospitalar. Rev Gaúch Enferm. 2014;35(2):79-85.

17. Guerra ST, Prochnow AG, Trevizan MA, Guido LA. O conflito no exercício gerencial do enfermeiro no âmbito hospitalar. Rev Latimoam Enferm. 2011;19(2):362-9.

18. Oderich C, Avelino LPRS, Queiroz MSF. Visão sistêmica interdisciplinar em grupo multiprofissional: estudo de caso em Foz do Iguaçu. RECC Rev Eletr Cienc CRA-PR [Internet]. 2015 [acesso em 2015 Nov 23];2(2):31-47. Disponível em: http://recc.cra-pr.org. br/index.php/recc/article/view/33

19. Gonçalves LA, Padilha KG. Fatores associados à carga de trabalho de enfermagem em Unidade de Terapia Intensiva. Rev Esc Enferm USP. 2007;41(4):645-52.

20. Altafin JAM, Grion CMC, Tanita MT, Festti J, Cardoso LTQ, Veiga CFF, et al. Nursing Activities Score e carga de trabalho em unidade de terapia intensiva de hospital universitário. Rev Bras Ter Intensiva. 2014;26(3): 292-8.

21. Abreu RMD, Gonçalves RMDA, Simões ALS. Motivos atribuídos por profissionais de uma Unidade de Terapia Intensiva para ausência ao trabalho. Rev Bras Enferm. 2014;67(3):386-93.

22. Machado DA, Louro TQ, Figueiredo NMA, Vianna LMA. Esgotamento dos Profissionais de Enfermagem: uma revisão integrativa sobre a Síndrome de Burnout em UTI. Rev Pesqui Cuid Fundam (Impr). 2012;4(4):2765-75.

23. Reader TW, Flin R, Cuthbertson BH. Communication skills and error in the intensive care unit. Curr Opin Crit Care. 2007;13(6):732-6.

24. Broca PV, Ferreira MF. Equipe de enfermagem e comunicação: contribuições para o cuidado de enfermagem.Rev Bras Enferm. 2012;65(1):97-103.

25. Gueudeville RM. Avaliação da comunicação entre a equipe multidisciplinar e do tempo de permanência 
na UTI, após a introdução do formulário de objetivos diários [dissertação]. Florianópolis: Universidade Federal de Santa Catarina; 2007.

26. Lazzari DD, Schmidt N, Jung W. Educação Continuada em Unidade de Terapia Intensiva na Percepção de Enfermeiras. Rev Enferm UFSM. 2012;2(1):88-96.

\section{Endereço para correspondência:}

João Dutra de Araujo Neto

Hospital Estadual Dirceu Arcoverde

Rua Rodrigues Coimbra, 1650

Bairro: Dirceu Arcoverde

CEP 64200-000 - Parnaíba - PI - Brasil

E-mail: joaodutrafisio@gmail.com 